\title{
A CASE OF THE SCHÖNLEIN-HENOCH SYNDROME WITH MYOCARDIAL NECROSIS
}

\author{
BY \\ M. A. LECUTIER \\ From the Department of Pathology, The Royal Infirmary, Derby
}

(RECEIVED FOR PUBLICATION APRIL 16, 1952)

The purpuras of Henoch and Schönlein have usually been described together under the term " anaphylactoid purpura." It is only in recent times, however, that papers on their pathology have appeared in which a definite lesion, which is common to both conditions, is described. This consists of an arteriolitis which, in severe cases, is necrotizing. It is now reasonably well established that the Schönlein-Henoch syndrome is due to a hypersensitivity reaction in which either a bacterial or a non-bacterial antigen, for example, an article of food, may be concerned (Gairdner, 1948).

It is the purpose of this communication to describe a case in which there was an opportunity to study the pathology after death. The case is of particular interest because much attention is being paid at present to the collagen diseases and because a cardiac lesion was found which apparently has not been described before in this syndrome.

\section{Case Report}

A. K. H., a boy of 11 years of age, was admitted to hospital complaining of a haemorrhagic rash and also of abdominal pain with vomiting. He had been perfectly well until six days before admission, when he had developed an exanthem on both legs, which in the first place consisted of vesicles. Two days before admission the joints had begun to swell, first the knees and then the elbows. There was nothing of note in the past history. On examination there were multiple small haemorrhagic macules on both legs, feet, and hands, and also many small discrete haemorrhagic vesicles on both legs and feet. Diffuse joint swellings were a feature, affecting both elbows, wrists, hands, and knees. The fauces were injected but the heart and lungs revealed no abnormal physical signs. The abdomen and central nervous system revealed no abnormality. The blood pressure was $142 / 98$ and the optic fundi presented narrow arterioles only. Two days later fresh petechiae appeared on the buttocks, and the next day a soft systolic apical murmur was noticed for the first time. Simultaneously several large purpuric patches appeared on the hands and feet, blood was passed per rectum, and the urine was noticed to be smoky. The blood pressure rose to
$145 / 105$ and the blood urea was $200 \mathrm{mg} . \%$. Penicillin therapy was instituted and it cleared the throat of haemolytic streptococci. Thereafter the patient's condition slowly deteriorated and he died approximately one month after admission.

Numerous investigations were performed. Albuminuria was present, together with varying numbers of casts, pus cells, and red cells, from the day of admission. Haemolytic streptococci were isolated from a throat swab two days after admission. The blood count on admission showed no anaemia but there was marked polymorphonuclear leucocytosis. Anaemia gradually developed and the leucocytosis persisted. The platelet count was never below normal. Bleeding on and clotting times were found to be normal on severa ? occasions. The cerebrospinal fluid and faeces likes wise were normal on repeated examination.

\section{Necropsy Report}

Externally there was moderate pallor and andsarca. Several dried haemorrhagic lesions were seen on the fingers and toes and one on the left forearm.

The brain presented generalized oedema of moderate degree. The lungs were oedematous and rubbery in consistency. The pericardium contained a large volume of clear, straw-coloured fluid. The $\dot{\sigma}$ heart was a little dilated and the valves were normal ; the myocardium was rather pale and there $\delta$ were a few scattered, small, hard nodules of $₹$ irregular outline in the wall of the left ventricle; $ᄋ$ the coronary arteries appeared normal. The liver $D$ presented a mild degree of passive congestion. Both kidneys were large and pale with broad, pouting cortices. The mucosa of the bladder was $\Omega$ haemorrhagic but otherwise normal. The small $N$ intestine presented several discrete haemorrhagic $\underset{\mathrm{N}}{\mathrm{N}}$ patches in its wall and the terminal 18 inches of the ileum were thus uniformly affected. The peritoneal cavity contained a fairly large volume of clear, straw-coloured fluid. None of the large arteries $\stackrel{?}{?}$ appeared abnormal in any way and all other 0 organs and parts appeared normal.

Histologically, sections from the spleen, the 
skin, the bladder, the ileum, the lungs, the kidneys, and the liver presented a periarteriolar infiltration with lymphocytes, histiocytes, polymorphs, and eosinophils, and, moreover, the arteriolar walls were tnemselves infiltrated by these cells, and in many cases there was in addition fibrinoid necrosis of the walls. In the case of the bladder and ileum, the lumina of some of these necrotic vessels were plugged with hyaline thrombus. As regards the heart, although several blocks and serial sections were examined, only a very few arterioles showed any sign of involvement and these were very small and showed no evidence of superadded thrombosis. The capillaries presented a similar perivascular infiltration in the case of kidneys, the liver and the skin, and there was patchy necrosis of the glomerular tufts of the kidney. Apart from the vascular lesions, the myocardium presented several areas of necrosis with calcification and some of these were extensive ; they corresponded to the hard nodules found at necropsy; none was found to be related to any involved arterioles, none of which in any case showed any evidence of thrombosis. In addition, several necrotic areas were seen without calcification, but these were not as

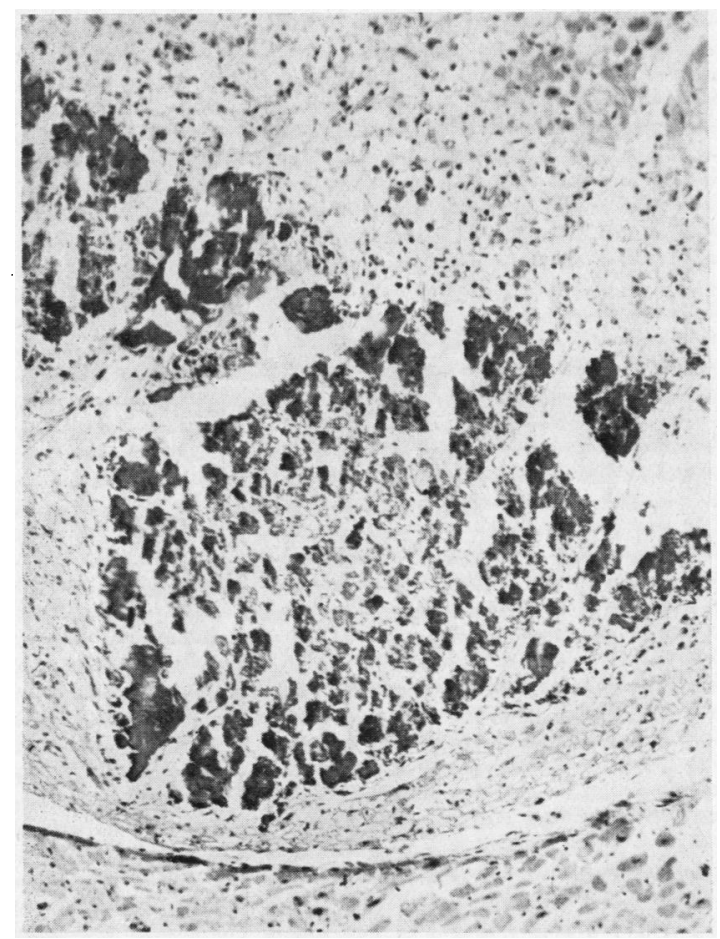

Fig. 1.-Photomicrograph $(\times 80)$ of area of myocardial necrosis with calcification. There is only a mild degree of surrounding cellular infiltration, the cells being practically all lymphocytes. Stained by haematoxylin and eosin. large as the calcified ones. Surrounding each lesion there was a mild degree of infiltration with small round cells only (Fig. 1). The kidneys presented the appearances of a subacute nephritis (Ellis type 1) with typical epithelial crescents ; moreover the stroma showed a mild degree of patchy infiltration with lymphocytes, eosinophils, and polymorphs. The liver showed a moderate degree of fatty degeneration. The appearances in the lungs were consistent with those of so-called uraemic pulmonary exudate (Doniach, 1947).

\section{Discussion}

In all essentials the case described is typical: clinically in that the three main symptoms, an exanthem, gastro-intestinal upset, and joint lesions, were present; aetiologically in that there was an original haemolytic streptococcal infection as a result of which a hypersensitivity mechanism probably operated ; and pathologically in that the basic lesion was an acute arteriolitis with perivascular infiltration (Spiegel, 1936; Miller and Daley, 1946 ; Gairdner, 1948).

There is little information in the literature concerning the histological appearances of the lesions in the gut. The typical lesion of necrotizing arteriolitis was found in this case and thrombosis was superadded (Fig. 2). Homb (1946) described a case in which typical lesions were found in the gut.

However, the areas of myocardial necrosis with calcification constitute the most interesting feature of this case. Apparently such a lesion has not been previously described in the Schönlein-Henoch syndrome and its pathogenesis is a matter of doubt. There would appear to be three lines of approach in attempting an explanation: (1) The lesion may be the result of ischaemia, resulting from necrotizing arteriolitis with thrombosis. (2) The lesion may represent a modified Aschoff body. (3) The lesion may be the result of the same mechanism as that producing the vascular lesions.

With regard to the first possible explanation, although this appears to be the most reasonable one, no practical support was forthcoming because no arteriolitis with thrombosis was found in the myocardium although several blocks and several sections were examined. Only a very few small arterioles showed any evidence of involvement in all these sections, none showed thrombosis, and none was related to the areas of necrosis described; moreover these areas of necrosis were large, such as could only be produced by blockage of fairly large vessels and would hardly escape detection in serial sections. The theory of ischaemia is therefore unacceptable. 


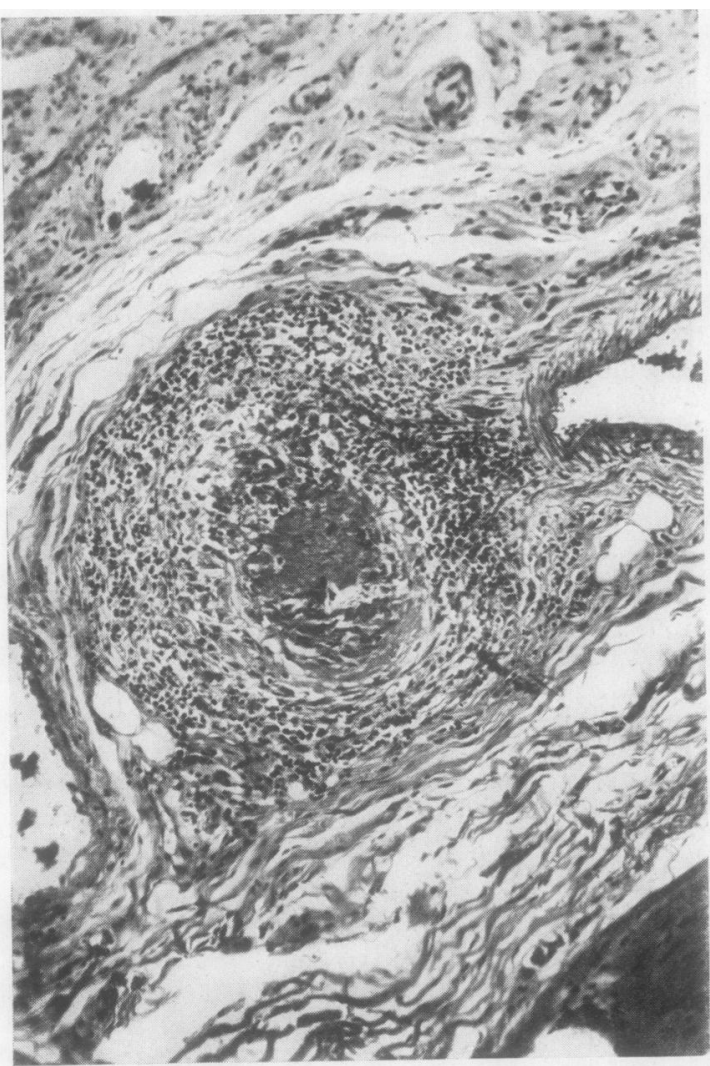

Fig. 2.-Photomicrograph $(\times 80)$ of an arteriolar lesion in the small intestine, showing heavy cellular infiltration of the vessel wall, the lumen being plugged by a thrombus. Stained by haematoxylin and eosin.

With regard to the second suggested explanation, it must be borne in mind that polyarteritis nodosa, and presumably its variant the Schönlein-Henoch syndrome, is closely allied to acute rheumatism. Thus both conditions are in all probability allergic diseases (Gairdner, 1948; Rich and Gregory, 1943), the vascular lesions of each are very similar, and fibrinoid necrosis occurs in each. That polyarteritis nodosa and rheumatic fever are closely allied is shown by Friedberg and Gross (1934), who described in detail four cases of polyarteritis nodosa associated with rheumatic fever. They believe in fact that rheumatic fever is a common cause of polyarteritis nodosa. One might therefore postulate that the necrotic myocardial lesion described represents a modified Aschoff body. The virtual absence of any surrounding inflammatory cells in the case of the lesion in the present case indicates, however, that the lesion is certainly not identical with the Aschoff body, but it may be argued that it represents the first stage of an Aschoff body before there has been adequate time for a cellular reaction to occur. The large size of $\stackrel{0}{\vec{*}}$ the lesions, together with the calcification make this explanation unlikely. That the lesion is a $\overrightarrow{\vec{c}}$ modified Aschoff body is therefore unacceptable.

As the first two explanations are unacceptable, $\frac{\mathrm{C}}{\mathrm{O}}$ although initially they appeared the most logical $\overline{\bar{\omega}}$. ones, one must accept the third explanation in $\overrightarrow{\mathbb{\Phi}}$ the absence of a better. Fundamentally there $ᄋ$ would appear to be no reason why an antigen- $ळ$ antibody reaction which affects the arterioles and $\overrightarrow{0}$ capillaries should not also affect the myocardium. If this is the correct explanation, it would, how- $\overrightarrow{-}$ ever, be expected that the lesion would have been $\frac{\Omega}{0}$ described before. It must be concluded that some? unknown additional factor is concerned and future observations may bring this to light. That an addi- $-\dot{\omega}$ tional factor may be concerned in antigen-antibody reactions is shown by Ahlström (1936) who pro-응 duced diffuse glomerulitis in rabbits sensitized to horse serum only after an injection of a smallz quantity of Staphylococcus toxin.

Finally one should consider briefly the relation of the Schönlein-Henoch syndrome to polyarteritis nodosa. Undoubtedly the lesions in these two conditions are essentially the same. Moreover the clinical resemblance between the two conditiogs is impressive; thus skin, joint, and intestim? lesions are all well described in polyarteritis nod (Spiegel, 1936 ; Miller and Daley, 1946). Gairdne? (1948) stresses the fact that capillaries are involved in the Schönlein-Henoch syndrome and arteries irg polyarteritis nodosa, arteriolar lesions being com $=$ mon to both. Certainly in this case arterioles anc capillaries only were involved, but this cannot be considered a fundamental difference between the two syndromes. Spiegel (1936) refers to capillar. lesions occurring in polyarteritis nodosa, and? moreover, the frequent occurrence of nephritis is polyarteritis nodosa is evidence of frequent capit lary involvement. It is suggested therefore that the two syndromes constitute the same condition, an $\vec{P}$ that whether the arteries or capillaries are involves depends on variations in the conditions of pathos genesis operating in any particular case.

Grateful acknowledgment is made to Dr. D. Wh Hubble, under whose care the patient was admitted, for access to the clinical details, and to Dr. G. R Osborn for his helpful advice.

\section{REFERENCES}

Ahlström, C. G. (1936). Acta path. microbiol. scand. Supp!. 29. Quoted in Hadfield, G., and Garrod, L. P. (1947). Rece

Advances in Pathology, Sth ed. London.
Doniach, I. (1947). Amer. J. Roentgenol.. 58, 620 .

Doniach, I. (1947). Amer. J. Roentgenol. S8, 620.

Friedberg, C. K., and Gross, L. (1934). Arch. intern. Med., 54, 170.

Gairdner, D. (1948). Quart. J. Med., 17,93.

Miller, H. G., and Daley, R. (1946). Quart. J. Med., n.s., 15, 255. Rich, A. R., and Gregory, J. E. (1943). Bull. Johns Hopk. Hosp., 70 239 .

Spiegel, R. (1936). Arch. intern. Med., 58, 993. 